\title{
DESENVOLVIMENTO DE UM SISTEMA PARA MONITORAMENTO E TELEOPERAÇÃO DE MÁQUINAS-FERRAMENTA CNC VIA INTERNET ADERENTE À INDÚSTRIA 4.0
}

\author{
L. E. S. Oliveira ${ }^{1}$; A. J. Álvares ${ }^{1 *}$ \\ ${ }^{1}$ Universidade de Brasília, Departamento de Engenharia Mecânica, 70910-900, Brasília-DF, Brasil \\ *alvares@alvarestech.com
}

\section{RESUMO}

As empresas vêm passando por constantes mudanças organizacionais a fim de se manterem competitivas. A indústria manufatureira investe fortemente em insumos como as tecnologias de informação e comunicação (TIC). Padrões como MTConnect e OPC-UA estão criando o caminho para o desenvolvimento da nova era industrial, este trabalho apresenta a arquitetura de um framework implementado na forma de um sistema cliente-servidor baseado na internet para o monitoramento e a teleoperação máquinas-ferramenta $\mathrm{CNC}$, e que apresenta atributos em conformidade com a "Indústria 4.0". A concepção do framework foi realizada através de uma abordagem metodológica que envolveu o uso de diagramas IDEF0 e a metodologia de Projeto axiomático para o refinamento do modelo. $\mathrm{O}$ trabalho de implementação computacional fundamentou-se na integração de servidores na forma de serviços através de um sistema na web, o CyberDNC.

\section{DEVELOPMENT OF A SYSTEM FOR MONITORING AND TELEOPERATION CNC MACHINE TOOLS THROUGH THE INTERNET ADHERENT TO INDUSTRY 4.0}

\begin{abstract}
Companies have been undergoing constant organizational changes in order to remain competitive. The manufacturing industry invests heavily in inputs such as information and communication technologies (ICT). Standards such as MTConnect and OPC-UA are creating the path to the development of the new industrial era, this paper presents the architecture of a framework implemented as an Internet-based client-server system for monitoring and teleoperation $\mathrm{CNC}$ machine tools,
\end{abstract}

and which features attributes in accordance with "Industry 4.0". The design of the framework was carried out through a methodological approach that involved the use of IDEF0 diagrams and the Axiomatic Design methodology for the refinement of the model. The computational implementation work was based on the integration of servers in the form of services through a web system, the CyberDNC.

KEYWORDS: Industry 4.0, MTConnect, OPC, CNC, teleoperation. 


\section{INTRODUÇÃO}

O mundo empresarial passa por constantes mudanças impulsionadas pela necessidade das organizações se manterem competitivas em um mercado global e responderem aos anseios de consumidores exigentes com demandas variadas e contínuas. Nesse contexto, as empresas estão vendo a necessidade de adequar os seus processos em estruturas que garantam uma produção mais eficiente, com mais qualidade, menores custos e tempos de ciclo reduzidos. No âmbito das empresas de manufatura os fatores de competitividade estão intimamente relacionados ao sistema de produção, envolvendo uma adequada gestão do chão-de-fábrica e uma clara visão sobre o seu estado operacional. Observando isso, a indústria manufatureira investe cada vez mais na integração de suas instalações físicas por meio das tecnologias de informação e comunicação (TIC) a fim de obter informações sobre o processo produtivo de forma detalhada, precisa e em tempo real, e com isso decisões possam ser tomadas, produtos sejam aprimorados e as expectativas individuais dos consumidores possam ser atingidas.

Atribui-se a Internet o papel de elemento-chave que permitirá uma integração e comunicação rápida entre pessoas e empresas. Em um passado recente a Internet tem sido empregada com sucesso em atividade diversas e importantes como a manutenção remota de equipamentos (Rockwell-Automation, 2001) e a tele-cirurgia conduzida através de um robô comandado remotamente por um cirurgião situado a quilômetros do paciente (Scientific-American, 2000). No âmbito da manufatura, acompanhando as mudanças nas estratégias de produção focadas no uso intensivo das tecnologias da informação, foram realizados uma série de estudos relacionados ao uso da Internet aplicado a manufatura remota e distribuída, implementando paradigmas como a E-manufacturing (manufatura eletrônica) (Álvares et al. (2002), Álvares et al. (2005), Álvares e Ferreira (2008), Benavente (2011)).

A E-manufacturing (e-Mfg) forma um ambiente de manufatura baseado em TICs, especialmente em tecnologias de rede, incluindo a Internet, utilizando métodos de trabalho vinculados a Collaborative e-Work (trabalho eletrônico colaborativo), empregado no ciclo de desenvolvimento de produtos que envolvem sistemas CAD/CAPP/CAM integrados (Lee, 2003, Nof, 2004). A Indústria 4.0 fundamenta-se na integração dos elementos da cadeia de valor através das tecnologias da informação e comunicação para o desenvolvimento das Smart Factories (fábricas inteligentes). Nessa perspectiva os sistemas para a Indústria 4.0 abrangem os sistemas de $e-M f g$, mas expandem o conceito de integração de informações para o nível de sensores e atuadores ao ter como base os Cyber-physical Systems (Sistemas Ciber-físicos, CPS) e Internet of Things and Services (Internet das Coisas e de Serviços).

Os CPSs e a introdução da Internet das Coisas e da Internet de Serviços (IoT e IoS) na indústria é o que caracteriza a era da industria 4.0 (Kagermann et al., 2013). Os sistemas Ciberfísicos representam a junção de sistemas embarcados, a Internet, dados e serviços disponíveis online (Mcdougall, 2014). Segundo McDougall (2014), CPSs criam as bases para a criação de uma Internet das Coisas, que combinada com a Internet dos Serviços forma o que é conhecido como Indústria 4.0. A Internet das Coisas é uma rede inteligente que conecta todas as coisas a Internet, a exemplo de sensores e atuadores, com a finalidade de permitir a comunicação entre esses dispositivos através de protocolos conhecidos, permitindo à identificação, o rastreamento, a localização, o monitoramento e o gerenciamento inteligente dessas entidades, Gubbi et al. (2013).

Os sistemas para a Indústria 4.0 estão fundamentados na integração de dados e serviços virtualmente localizados que estão vinculados aos recursos físicos da manufatura, através de uma rede inteligente $(\mathrm{Cloud})$. Essa integração é garantida através do uso de padrões de referência. A adoção desses padrões são apontadas por Kagermann et al. (2013) como um requisito para a adequada implementação das tecnologias que compõem a quarta revolução industrial. Nesse nível, 
destacam-se padrões de conectividade como o OPC para a internet, o OPC-UA (Open process control united architecture), e o mais recentemente desenvolvido, MTConnect.

O MTConnect é um padrão aberto (royalty-free) e foi desenvolvido com o propósito de se tornar um protocolo que promovesse a integração de máquinas CNC (Comando Numérico Computadorizado) (Sobel, 2010). O OPC-UA foi desenvolvido pela OPC Foundation e representa uma evolução do OPC clássico, baseado em tecnologia COM/DCOM, para formar um padrão de interoperabilidade independente de plataforma para a troca de dados entre o chão-de-fábrica e os níveis de decisão estratégicos das organizações (Mahnke et al., 2009). Enquanto o MTConnect facilita a conexão entre máquinas-ferramenta $\mathrm{CNC}$ e outros equipamentos em rede para coleta de dados de fabricação, o OPC-UA promove a interoperabilidade necessária para a comunicação de dados em toda a planta (Albert, 2015).

A teleoperação remota é uma aplicação vinculada à atividades eletrônicas (e-activities), a exemplo da e-manufacturing (Nof, 2004), e que neste é relacionada ao contexto das fábricas inteligentes como um recurso para garantir onipresença e informação de realimentação para auxiliar na atuação sobre o processo de fabricação, que neste caso é a manufatura por usinagem.

Com isso, o presente trabalho propõe o planejamento e a implementação de um framework para monitoramento e teleoperação de máquinas-ferramenta $\mathrm{CNC}$, tendo como elemento de teste e validação um centro de torneamento $\mathrm{CNC}$ da marca Romi modelo Galaxy $15 \mathrm{M}$, provido com controlador Fanuc 18i-Ta. A implementação tem como resultado um sistema com arquitetura cliente/servidor baseada na Web (CyberDNC), combinado com elementos aderentes a Indústria 4.0, como um serviço MTConnect para acesso a dados de CNC, serviço OPC via Web para interação com a máquina através de funções de PMC/PLC (Programmable machine control/Programmable logic controller). Além dessas funções, há os serviços de comando remoto via HTTP e programas CGI (Common gateway interface), e monitoramento por imagem através de um serviço de streaming de vídeo via internet.

\section{TRABALHOS CORRELATOS}

Há uma significativa produção bibliográfica relacionada à concepção de aplicações relacionadas a conectividade de dispositivos industriais envolvendo a especificação OPC. Dentro desse universo estão os trabalhos voltados para a proposição de arquiteturas de monitoramento e controle distribuído do chão-de-fábrica baseado no acesso OPC através da internet. Com base nesse paradigma, trabalhos vêm sendo desenvolvidos explorando as características da emergente especificação da OPC Foundation, o OPC-UA.

O MTConnect é um padrão recente, mas a sua versatilidade e os benefícios potenciais gerados a partir de seu uso tornam essa especificação objeto de intensa investigação dentro do meio acadêmico e industrial. Li e Hu (2008) utilizam a comunicação DCOM entre um cliente OPC-DA e o servidor OPC-DA, os dados transferidos para o cliente OPC-DA são convertidos em formato XML para que os dados possam ser acessados através da Internet por um cliente XML-DA que se comunica com um servidor XML (servidor web) para obter esses dados.

Sahin e Bolat (2009) utilizam uma arquitetura distribuída para OPC (DOPC) para realizar o monitoramento e controle remoto de diferentes dispositivos baseado na web. Arquiteturas de controle local desenvolvidas em vários pontos possibilita a comunicação uns com os outros e com um ponto de controle remoto em uma página web dinâmica construída usando Active Server Pages (ASP). Fernandes et al. (2009) utilizam uma tecnologia de transporte com a função de gateway (OPC Server para HTTPs), chamada CyberOPC, para elaborar uma arquitetura para executar o 
ajuste remoto de sistemas de controle industrial usando a Internet, cumprindo requisitos de segurança e desempenho aceitáveis.

Vijayaraghav e Dornfeld (2010) apresentam um sistema para o monitoramento do consumo de energia desenvolvido usando MTConnect e um sistema de motor de regras e processamento de evento complexo (CEP), para suportar o racionamento de dados e processamento de informações. Esses dados e informações são armazenados na nuvem e vão alimentar análises de alta frequência de dados em series temporais. No trabalho de Silva et al. (2012), o padrão MTConnect foi usado para a aquisição de dados de CNC em um sistema de supervisão baseado na Web para operações de esmerilhamento. Michaloski et al. (2013) propõem uma arquitetura de um sistema de manufatura para prover, através da Web, dados em tempo real e estatísticas para a garantia da qualidade, baseado na integração de duas especificação abertas: MTConnect e QMResults (Quality Measurement Results).

Chen et al. (2017) propuseram um sistema de monitoramento de máquinas para CNCs baseado em MTConnect descrevendo a implementação do protocolo. A implementação inclui o estabelecimento de um modelo de informação para CNC em conformidade com o padrão MTConnect, coletando e processando dados de CNCs através do adaptador, estabelecendo comunicação com o Agente MTConnect para este interagir com a aplicativo de monitoramento através de requisições HTTP. O sistema é avaliado com um $\mathrm{CNC}$ real, e os resultados mostram a viabilidade prática do MTConnect para o monitoramento do dispositivo.

Verma et al. (2017) apresentam o monitoramento remoto em tempo real de um compressor de ar alternativo baseado no protocolo padrão MTConnect para transmitir dados relacionados ao compressor para os clientes ou aplicações. Os dados de acústica e vibração do compressor de ar são fornecidos a dois clientes (navegador web e MS Excel) por meio de um Agente MTConnect dedicado. Os dados obtidos no final do cliente possuem uma marcação de data/hora que notifica o cliente sobre o instante em que esse dado particular foi adquirido do dispositivo.

\section{METODOLOGIA}

A proposta do trabalho trata de um sistema cliente-servidor que envolve diferentes módulos conectáveis através da internet, sendo classificado como um sistema complexo. Inicialmente, utilizou-se a modelagem IDEF0 (Integration Definition language for function modeling) que fornece uma visão geral dos múltiplos níveis de detalhamento das atividades que compõem o framework, enfatizando as principais entradas, saídas, os recursos utilizados (mecanismos) e regras (controles) do framework. Esses diagramas auxiliam no levantamento das necessidades dos usuários, que são importantes para o projeto axiomático, atuando como uma entrada para esta outra metodologia.

A técnica de Projeto Axiomático (AD - Axiomatic Design) ajuda a modelar os detalhes do projeto pela especificação dos parâmetros que compõem os seus módulos até a geração da arquitetura detalhada do sistema, juntamente com a sua sequência de implementação.

\subsection{Modelagem IDEF0}

A utilização do IDEF0 dá a arquitetura do framework um aspecto mais genérico com a finalidade de permitir que a mesma atue como uma referência geral para futuros projetos. Nesse sentido, o nível principal da modelagem (A0), representado na Figura 1, leva o nome de framework. 


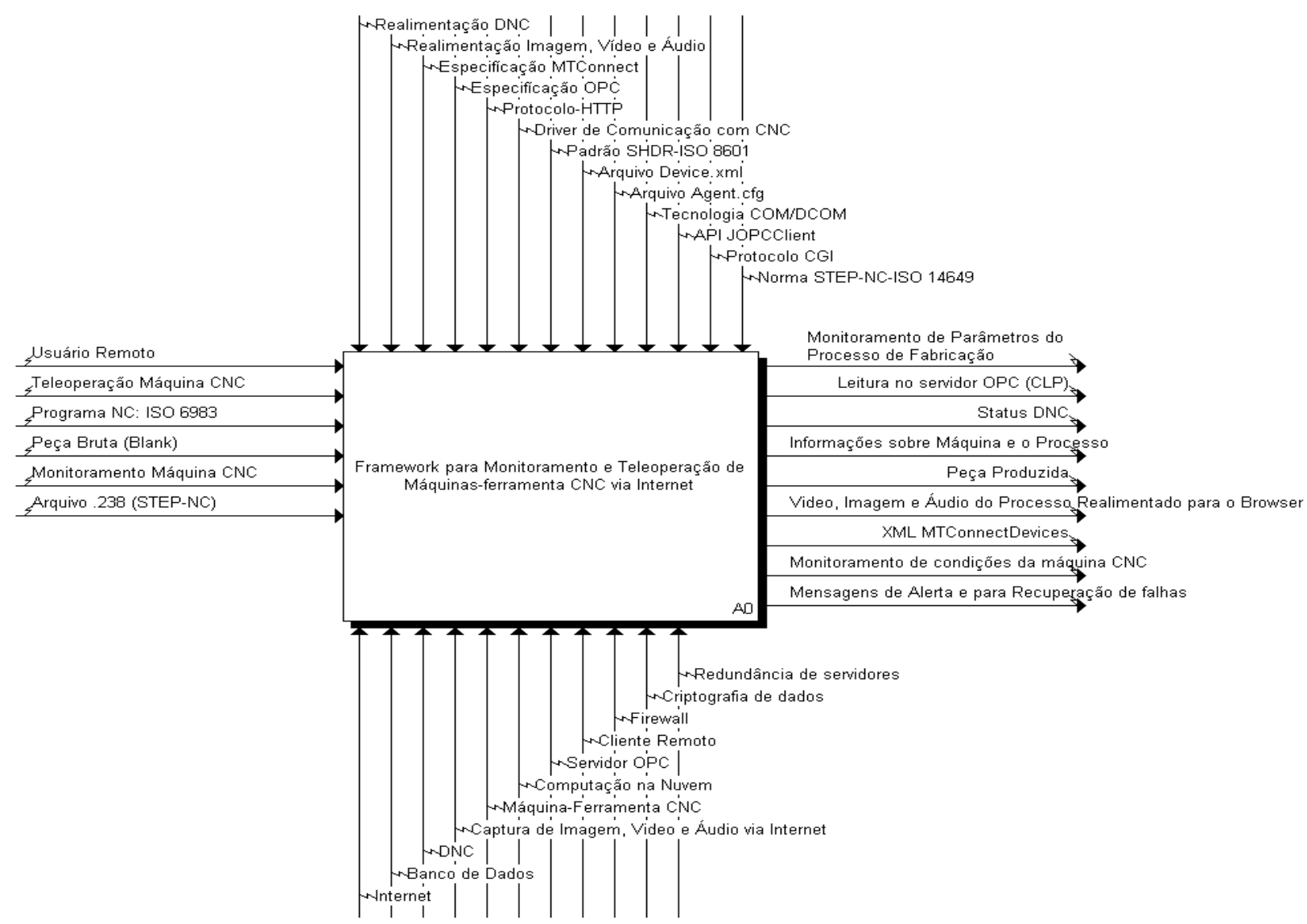

Figura 1: Modelagem IDEF0 do framework: nível A0.

Fonte: Elaborado pelos autores (2017)

Essencialmente, as entradas de um sistema projetado a partir dessa arquitetura contam com a atividade de monitoramento, a teleoperação, usuários situados remotamente, a peça bruta (blanck), que é uma entrada para a máquina-ferramenta onde está situado o $\mathrm{CNC}$, e o programa $\mathrm{NC}$, que contem os comandos de fabricação de uma peça. Foi incluído nas entradas do modelo um arquivo ".238" como uma proposta para potenciais trabalhos utilizando controladores compatíveis com o padrão STEP-NC.

As saídas do framework envolvem o produto dos serviços de monitoramento da máquina e do processo de fabricação, dos serviços de teleoperação, supervisão e monitoração visual.

Os mecanismos da arquitetura, representados pelas entradas da parte inferior da atividade (Fig. 1), possuem como uma de suas funções, posicionar o projeto no contexto dos sistemas para a Indústria 4.0, através da inclusão de recursos como: internet, computação na nuvem, bancos de dados (na nuvem), imagens de vídeo do chão-de-fábrica em tempo real.

Os controles são representados pelas especificações, como a OPC (OPC-UA) e o MTConnect, protocolo HTTP, e APIs (Application Protocol Interfaces) sugeridas como as principais para possibilitar a comunicação com os módulos servidores.

A decomposição do nível A0 resulta na estrutura básica do sistema, que é formada pelo conjunto de clientes e servidores. A Figura 2 ilustra à esquerda a atividade A1 que reúne os clientes web dos servidores, estejam eles na forma de aplicações acessíveis por meio de um browser de internet ou mediante uma aplicação para dispositivos móveis. A atividade à direita representa os servidores que compõem a plataforma como: teleoperação, monitoramento e supervisão. 


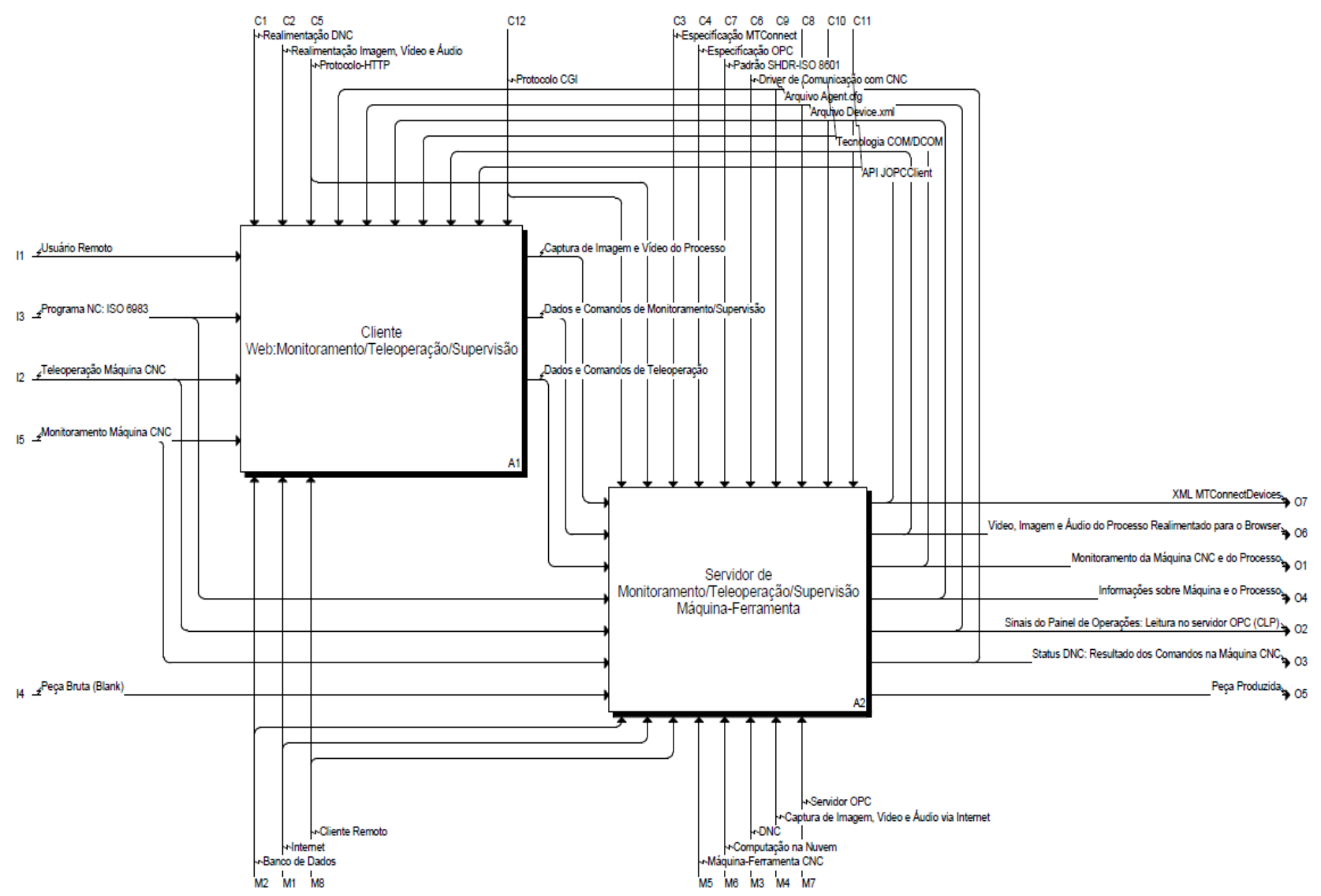

Figura 2: Grupos de clientes e servidores da arquitetura.

Fonte: Elaborado pelos autores (2017)

Baseado no diagrama da Figura 2 é pertinente destacar que na nova era da indústria os sistemas devem ser independentes de plataforma pela disponibilização de dados e informações em formatos universais, os clientes dessa plataforma devem ter acesso à internet por meio de um software navegador, para atividades de teleoperação, monitoramento e supervisão, ou por meio de aplicações para dispositivos móveis tipo smartphone e tablet, com funções de monitoramento/supervisão.

A Figura 3 mostra a estrutura e as comunicações dos servidores do framework. Na arquitetura proposta o monitoramento é realizado pelo serviço designado como Monitoramento/Supervisão (A21), que tem como insumos principais dados e comandos que formam basicamente requisições web. As saídas dessa atividade envolvem dados de supervisão e informações relacionadas ao status da máquina e do processo. A atividade A22, servidor WebCam, que provê serviços streaming de vídeo e áudio, é parte dos recursos de teleoperação. Por sua vez, O servidor WebCNC (A23) está diretamente vinculado ao CNC da máquina-ferramenta. Este serviço concentra os algoritmos que fornecem os comandos associados a funções de DNC que são executadas no controlador da máquina.

O serviço de Monitoramento/Supervisão (A21) ainda é subdividido em servidor de monitoramento MTConnect (A211) e o servidor OPC de supervisão via Internet, OPCWeb (A212), conforme mostra a Figura 4. O servidor MTConnect efetua o monitoramento principalmente através de streaming de dados de fabricação em formato XML, com a estrutura de dados definida pelo esquema MTConnectDevices. O servidor OPCWeb recebe entradas na forma de dados e comandos para requisição de dados de saída com o status recente de parâmetros de PLC ou para alterar esses parâmetros e efetuar o acionamento de controles da máquina-ferramenta CNC. 


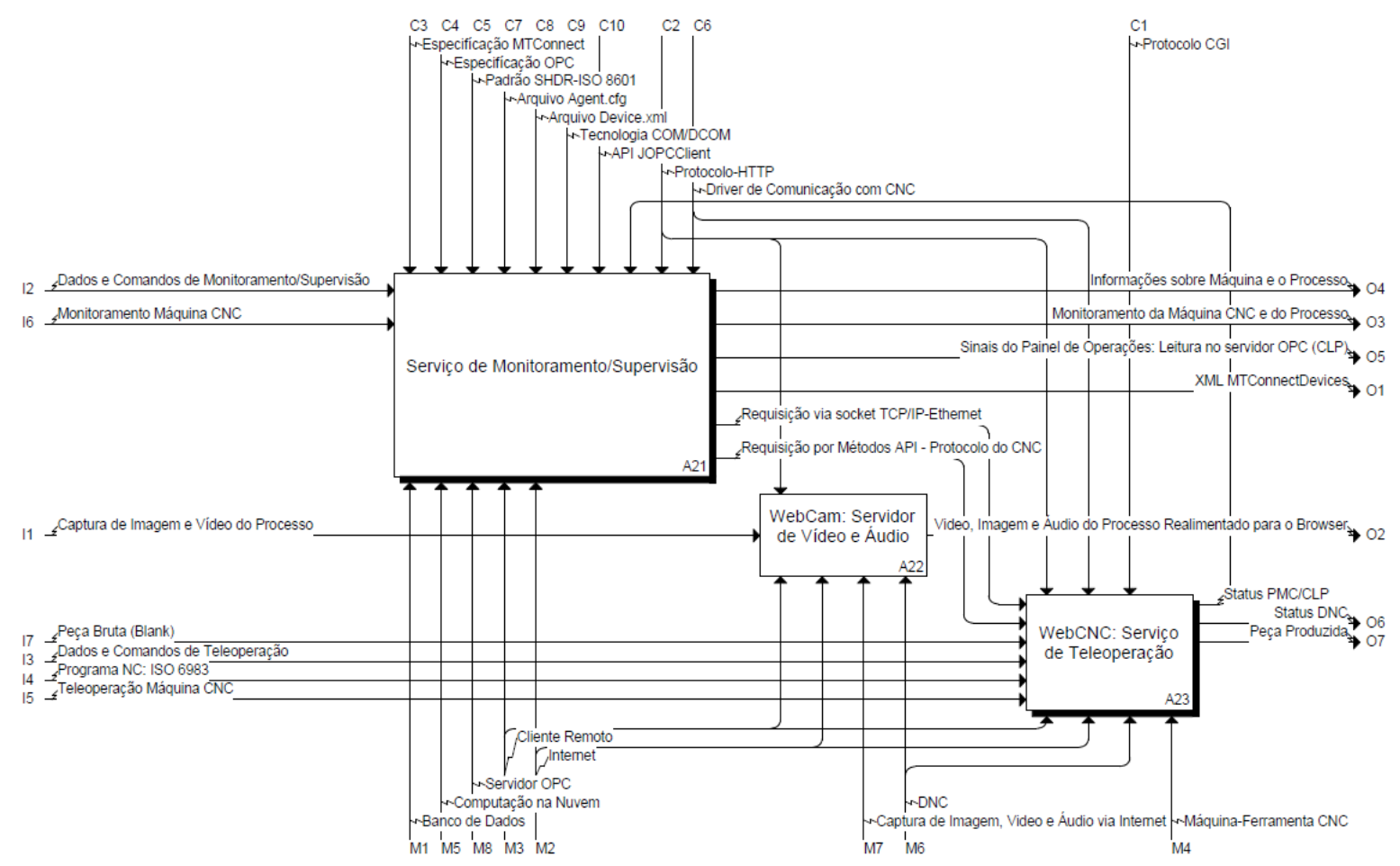

Figura 3: Atividades A21, A22 e A23: servidores da arquitetura.

Fonte: Elaborado pelos autores (2017)

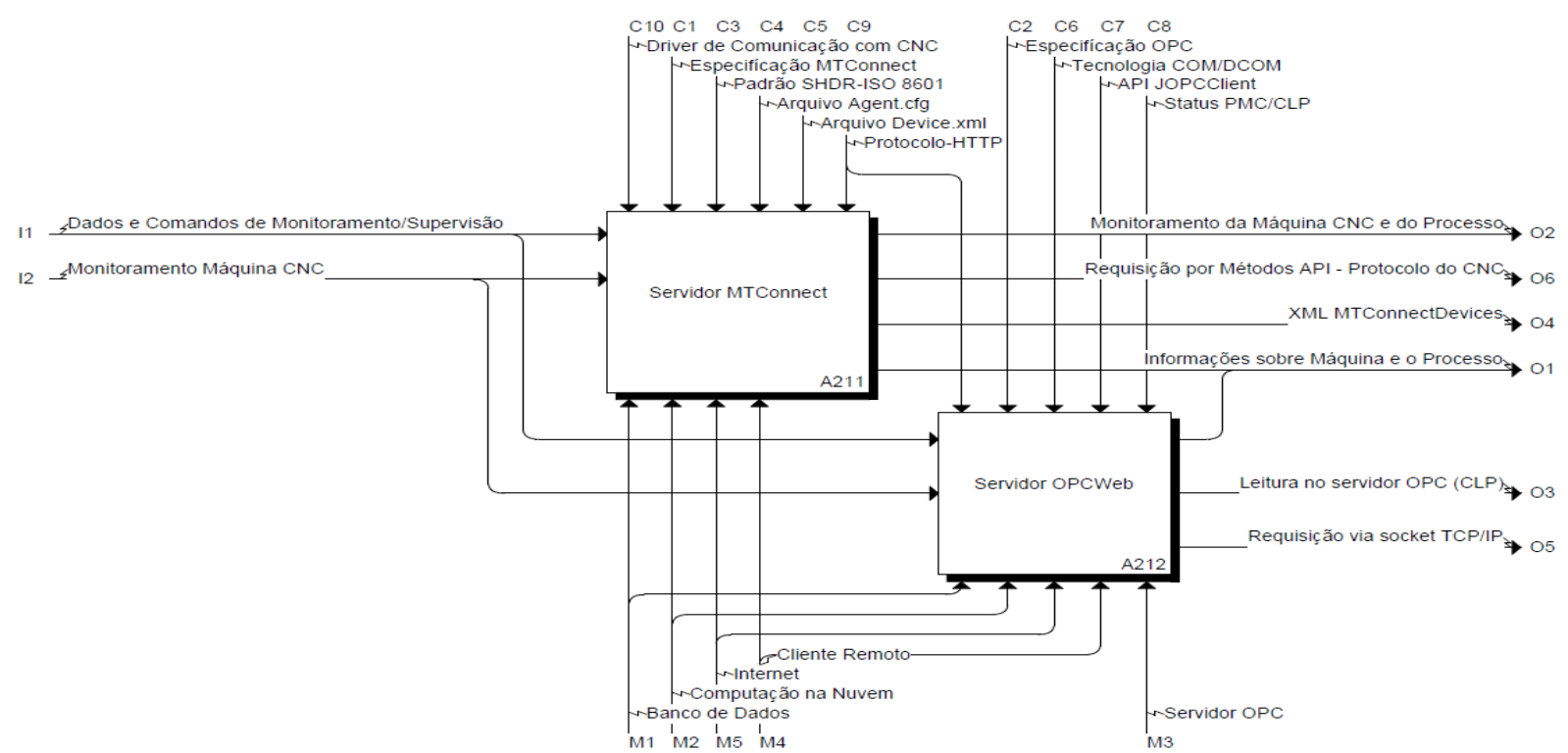

Figura 4: Atividades A211 e 212: Servidor de Monitoramento/Supervisão.

Fonte: Elaborado pelos autores (2017)

O servidor MTConnect proposto ainda é dividido em Adaptador e Agente. O Adaptador comunica-se com o controlador através da API fornecida pelo fabricante do CNC, e fornece dados de fabricação para o Agente. Este estrutura esses dados em formato XML, que é compreensível pelas aplicações cliente na internet. A especificação OPC-UA (OPC Unified Archtecture) também é apontada como um dos protocolos que irão ajudar a construir o caminho para o pleno desenvolvimento da Indústria 4.0 (Albert, 2015). No entanto, ainda é uma especificação em 
desenvolvimento, com um número de referências reduzido em termos de projetos acadêmicos e com soluções comerciais ainda nas primeiras versões e dependente de licenciamento para uso. Como alternativa, é proposto o servidor OPCWeb que adapta o software servidor OPC-DA clássico para ser acessado via internet por meio de um software middleware com a função de gateway entre o servidor OPC e o cliente final localizado na Web.

Como trata-se de uma arquitetura geral de referência, os mecanismos "banco de dados" e "computação na nuvem", para o servidor de monitoramento e supervisão, representam apenas propostas que não fazem parte dos elementos implementados. A necessidade de incluir esses recursos está relacionada ao fato de os projetos de plataformas no âmbito da Indústria 4.0 terem como estratégico uma infraestrutura baseada na nuvem e em bases de dados robustas que auxiliam na produção dinâmica de conhecimento sobre o ambiente de manufatura.

\subsection{Implementação do Projeto Axiomático}

A definição do modelo funcional permitiu identificar os fluxos de informações entre as funções e atividades do framework, pela identificação dos parâmetros de entrada, das saídas, dos mecanismos (recursos) e dos controles (regras). Essas informações, juntamente com levantamento bibliográfico envolvendo temas como projeto axiomático de software (Kim et al. (1991), Suh e Do, (2000), Suh (2001), Togay et al. (2012)), manufatura eletrônica (Álvares (2005), Benavente (2007), Álvares e Ferreira (2008)) e as aplicações para a manufatura do futuro e fábricas inteligentes (Shiet al. (2011), Kagermann et al. (2013), Monostori (2015)), ajudaram a produzir as principais necessidades para os potenciais usuários e as restrições dos sistemas projetados a partir dessa arquitetura. Esses parâmetros são abstraídos em um conjunto de pares (módulos) formado por Requisitos funcionais (Functional Requirements, FR) e o seus correspondentes Parâmetros de Projeto (Design Parameters, DPs). Um módulo corresponde ao mapeamento de uma entidade do domínio funcional de projeto (FR) em uma entidade no domínio físico (DP).

Essa metodologia também fornece a sequência de execução do projeto. Durante o processo de projeto, grupos de módulos correspondem aos componentes da arquitetura de detalhada que, neste trabalho, representam os diferentes servidores e o cliente do sistema.

Os módulos de software da arquitetura sistema com a necessidade de serem programados em linguagens orientadas a objeto, são tratados paralelamente mediante o modelo $\mathrm{V}$ e abordagem ADooSS (Axiomatic Design of Object-Oriented Software Systems), que gera como resultado a estrutura de classes do software, formada pelo conjunto de módulos.

O projeto tem como requisito funcional fundamental (FR0) a proposta de uma ferramenta para monitoramento e operação remota de máquinas-ferramenta $\mathrm{CNC}$, com elementos técnicos e conceituais aderentes a Indústria 4.0. O Parâmetro de Projeto (DPO) correspondente é "um framework baseado na Web para monitoramento e teleoperação de máquinas-ferramenta CNC". Movendo-se para o próximo nível na hierarquia de projeto, o parâmetro de projeto principal (DPO) é refinado nos requisitos funcionais definidos no Quadro 1, que também apresenta os mapeamentos desses requisitos em Parâmetros de Projeto. Esse mapeamento entre os domínios funcional e físico de primeiro nível resultou na matriz da Figura 5.

Nesse mapeamento de primeiro nível (FRx/DPx) verificou-se um significativo grau de desacoplamento, ou seja, há uma maior independência dos requisitos funcionais (Axioma 1) em relação aos parâmetros de projeto que o realizam. Mas percebe-se que nem todos os $F R s$ da matriz estão associados a um único $D P$ correspondente, $F R 2$ possui uma relação com $D P 3$, e $F R 4$ com $D P 1$. Durante o processo de abstração para a definição dos parâmetros de projeto concluiu-se haver uma relação entre a interatividade com controlador da máquina $(F R 2)$ com a construção de uma 
interface gráfica estruturada (DP3). Observou-se uma correlação entre manter dados sobre o processo em banco de dados $(F R 4)$ e a função de monitorar o processo via Internet (DPl). No entanto, com base em Suh (2001), um projeto aceitável, os parâmetros de projeto (DPs) e os requisitos funcionais (FRs) estão relacionados de tal forma que um $D P$ específico pode ser ajustado para satisfazer um $F R$ sem afetar outros. Com isso, os mapeamentos $F R$ × $D P$ fora da diagonal poderiam ser suprimidos sem afetar significativamente os mapeamentos posteriores.

Quadro 1: Mapeamento de primeiro nível, FRx em DPx

\begin{tabular}{|l|l|l|}
\hline $\mathbf{x}$ & $\mathbf{F R x}$ & DPx \\
\hline 1 & $\begin{array}{l}\text { Monitorar o processo de fabricação } \\
\text { remotamente }\end{array}$ & $\begin{array}{l}\text { Funções para monitorar o processo através da } \\
\text { Web }\end{array}$ \\
\hline 2 & Interatividade com o controlador da máquina & $\begin{array}{l}\text { Funções para configuração e comando da } \\
\text { máquina via Internet }\end{array}$ \\
\hline 3 & Interface intuitiva com o operador remoto & Interface gráfica estruturada \\
\hline 4 & $\begin{array}{l}\text { Manter dados sobre o processo de fabricação } \\
\text { em banco de dados }\end{array}$ & $\begin{array}{l}\text { Módulo de software vinculado a BD para } \\
\text { armazenar dados do processo }\end{array}$ \\
\hline
\end{tabular}

Fonte: Elaborado pelos autores (2017)

\begin{tabular}{|c|c|c|c|c|}
\hline DR & DP1 & DP2 & DP3 & DP4 \\
\hline FR1 & $x$ & & & \\
\hline FR2 & & $x$ & $x$ & \\
\hline FR3 & & & $x$ & \\
\hline FR4 & $X$ & & & $x$ \\
\hline
\end{tabular}

Figura 5: Decomposição de primeiro nível.

Fonte: Elaborado pelos autores (2017)

No projeto axiomático os parâmetros de projeto são decompostos em requisitos de funcionais filhos que geram novos parâmetros de projeto filhos, formando níveis de FRs e DPs em um procedimento de zigzagging entre o domínio funcional e físico do projeto, processo que é interrompida quando atinge-se um "nível folha", quando a partir dos requisitos de funcionais são abstraídos parâmetros de projeto definitivos. Os grupos de módulos gerados ao final desse processo desse processo de decomposição formam os subsistemas que compõem a estrutura do produto de projeto.

Finalizado o processo de decomposição e eliminadas as inconsistências das matrizes de projeto dos subníveis (DM1.x, DM2.x, DM3.x e DM4.x) da hierarquia de requisitos funcionais (FR) e parâmetros de projeto (DP), obtém-se a Matriz de projeto (Design Matrix, DM) completa. Esta matriz corresponde à junção das matrizes resultantes da decomposição dos parâmetros de projeto do Quadro 1.

A matriz de projeto completa é rearranjada a fim de aproximar os módulos com maior afinidade em termos de similaridade tecnológica ou finalidade dentro do projeto. O resultado dessa tarefa é representado pela matriz da Figura 6. 


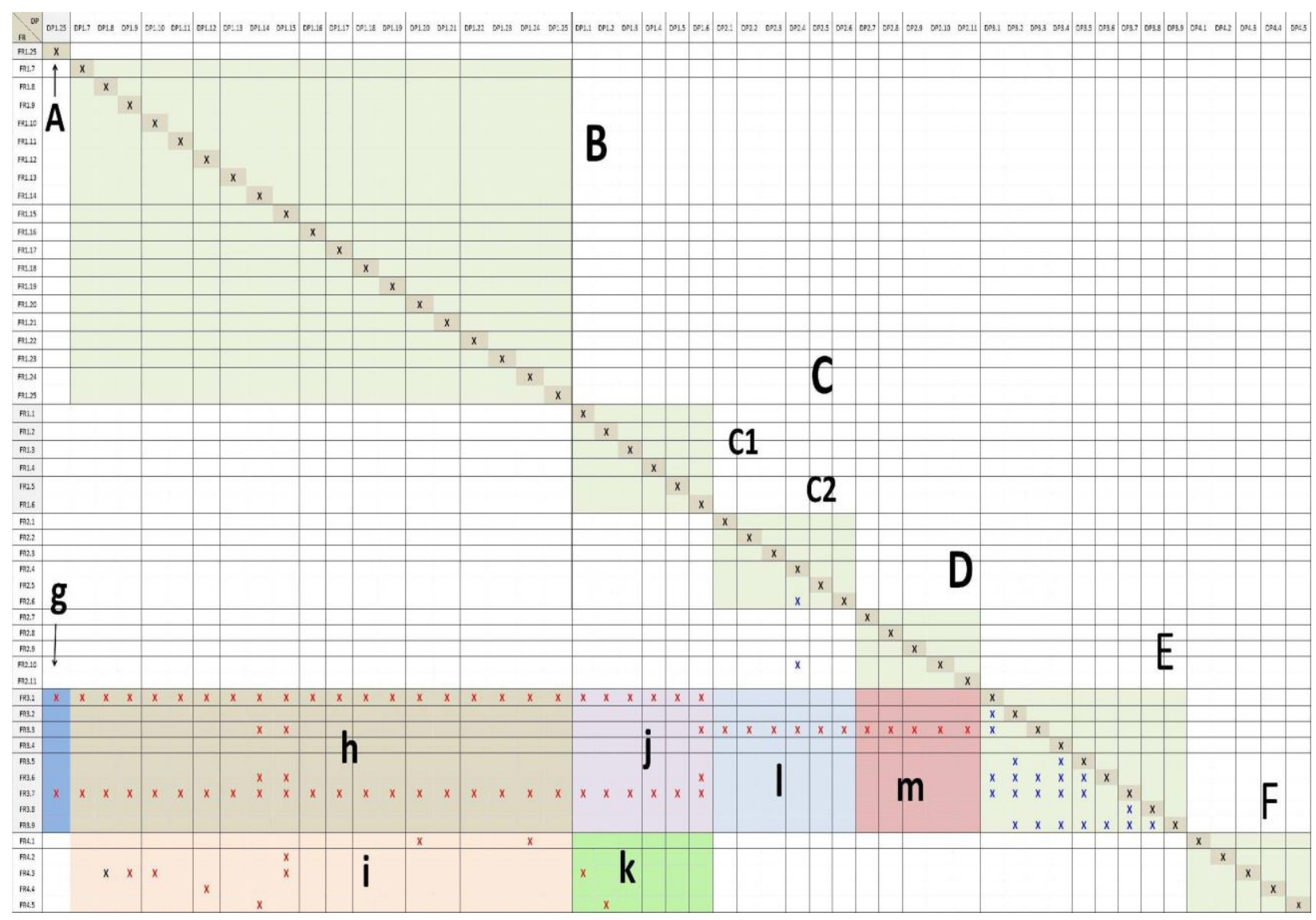

Figura 6: Matriz de Projeto Completa rearranjada.

Fonte: Elaborado pelos autores (2017)

Através da matriz obtida (Fig.6) é possível identificar nos módulos da matriz os serviços que compõem a arquitetura do sistema gerado. Dessa forma, foram extraídos os seguintes grupos de módulos:

(A): Módulo do servidor de streaming de vídeo (WebCam - Graco/UnB), que não foi programado no âmbito desse trabalho, mas foi selecionado para implementação.

(B): Parte do Servidor de Monitoramento, que emprega o padrão MTConnect. Na matriz é representado pelo grupo de módulos (M1.7 - M1.25) que formam a principal classe do software Adaptador (FocasGateway), que tem a função de fazer a comunicação do Adaptador com o CNC da máquina através da API desenvolvida pelo fabricante CNC.

(C) : Compõe servidor de OPCWeb e é constituído por módulos de leitura (C1) e escrita (C2) em parâmetros de CLP da máquina-ferramenta.

(D): Parte do serviço de comando remoto que utiliza protocolo DNC do controlador da máquina para o envio de comandos remotamente ao CNC.

(E): Módulos que caracterizam o Cliente Web para monitoramento e teleoperação.

(F): Classe com função de middleware para a persistência em banco de dados de informações capturadas durante o monitoramento/supervisão do processo.

(G): Funções para vincular o servidor de streaming de vídeo à interface Web cliente.

(H): Módulos para a comunicação entre Adaptador do CNC e o Cliente MTConnect (função do Agente MTConnect). 
(I): Módulos que vinculam os serviços de streaming de dados MTConnect aos métodos para salvar dados de parâmetros de status da máquina em banco de dados.

(J): Módulos que fazem parte do servidor OPCWeb com função de middleware para associar funções de leitura no servidor (CLP da máquina) ao cliente Web.

(K) Módulos que vinculam os serviços de supervisão OPC aos métodos para salvar o status de parâmetros de CLP da máquina em banco de dados.

(L) Módulos que fazem parte do servidor OPCWeb com função de middleware para associar funções de escrita no servidor (CLP da máquina) ao cliente Web.

(M) Módulos representando os programas para o envio de comandos DNC para o controlador da máquina através de mecanismos de acesso da Internet (CGI).

Com a matriz de projeto completa também é possível definir a sequencia de execução do projeto baseado na ordem dos caracteres alfabéticos. Um dos resultados da implementação do projeto axiomático é a arquitetura do sistema ou produto projetado, que pode ser mapeado em um esquema detalhado como na Figura 4.

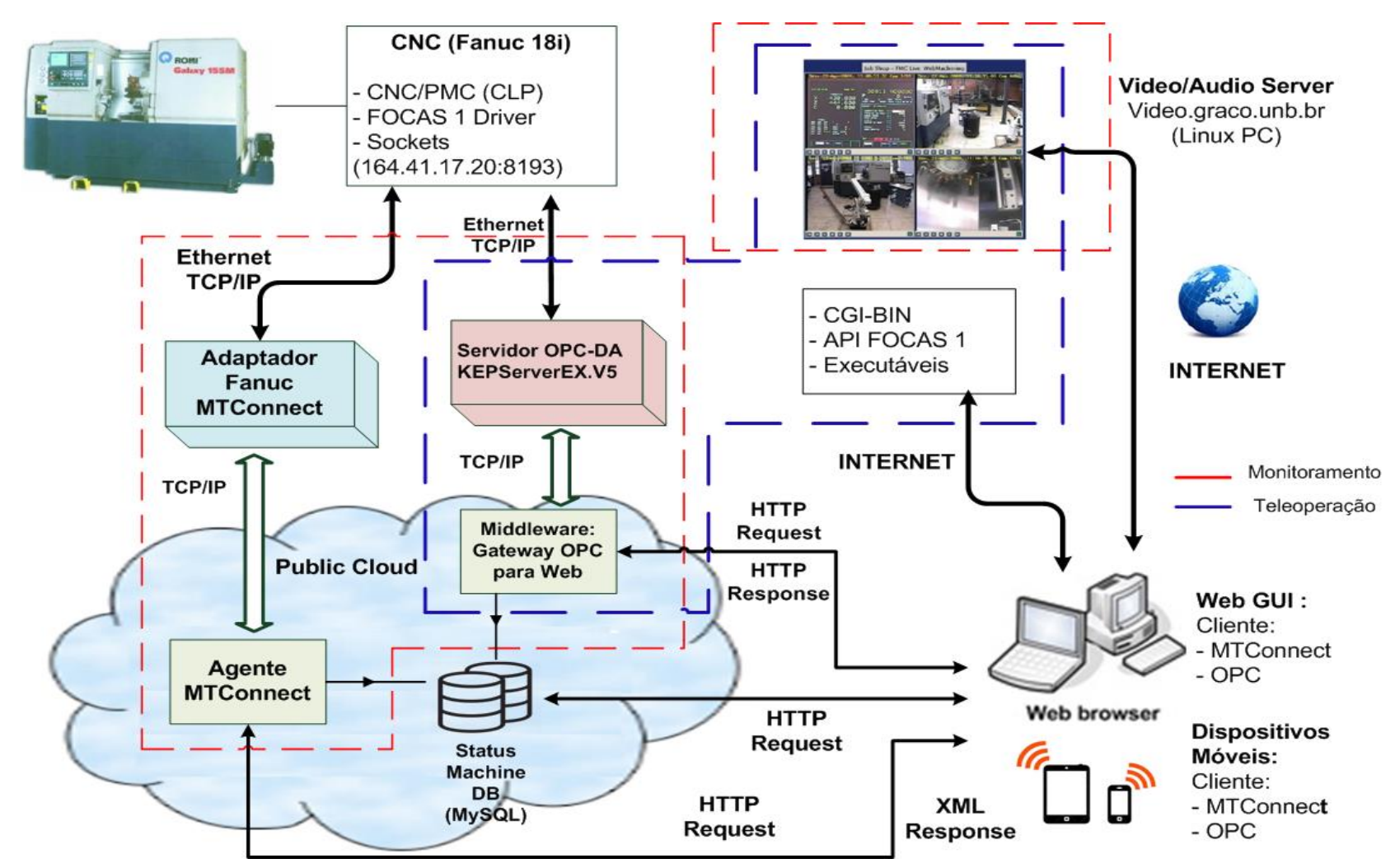

Figura 4: Arquitetura proposta para o sistema.

Fonte: Elaborado pelos autores (2017)

Na Figura 4 estão presentes os elementos definidos pelos módulos da matriz de projeto completa e as suas comunicações. Para a implementação não foram incluídos os elementos definidos como proposta após o processo de projeto da metodologia utilizada, que incluía mecanismos de computação na nuvem com banco de dados para atividades de monitoramento e supervisão, associados a localização física do processamento do Agente MTConnect e do middleware do servidor OPCWeb. No entanto, os elementos incluídos fornecem a estrutura básica de um sistema associado à estratégia da Indústria 4.0. 
Os elementos de software identificados na definição da arquitetura como subsistemas programáveis e exequíveis através de linguagens de programação orientadas a objeto foram tratados através de uma abordagem acessória conhecida como Projeto Axiomático de Sistemas de Software Orientados a Objeto (Axiomatic Design of Object-Oriented Software Systems, ADo-oSS) em que um dos principais resultados é o mapeamento dos módulos da matriz em diagramas de classes. A Figura 5 ilustra o diagrama de classes obtido a partir do mapeamento dos módulos associados ao Adaptador MTConnect na matriz de projeto completa.

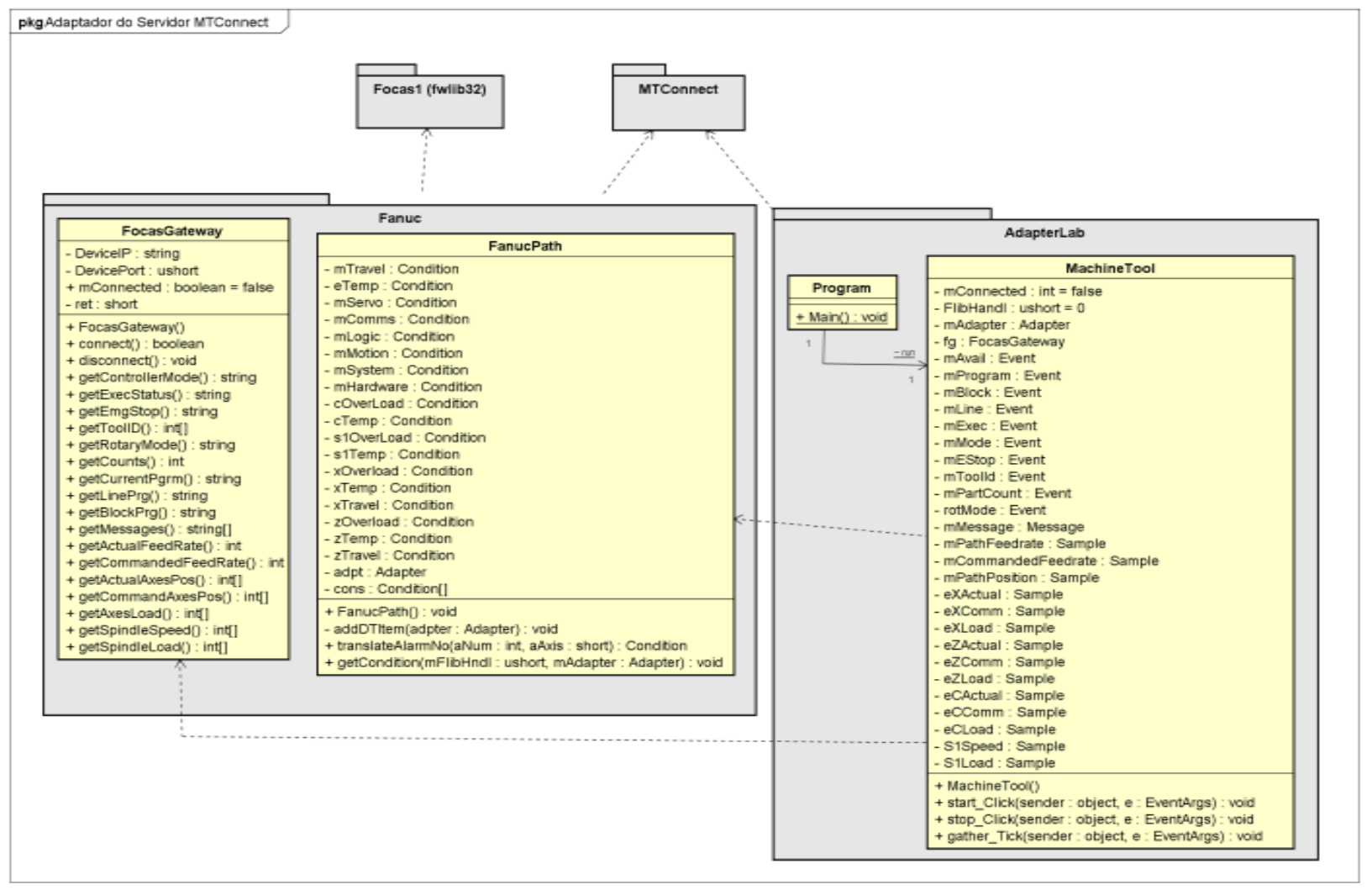

Figura 5: Estrutura de Classes do Adaptador Fanuc-Focas1.

Fonte: Elaborado pelos autores (2017)

\section{VALIDAÇÃO, RESULTADOS E DISCUSSÃO}

O sistema projetado possui uma arquitetura Cliente-Servidor com foco na integração de serviços baseados em padrões aderentes ao paradigma da Indústria 4.0, como o MTConnect e o OPC para internet.

Os serviços de monitoramento e supervisão baseiam-se essencialmente na aquisição de dados através de um servidor MTConnect, composto do servidor do CNC, software Adaptador e software Agente; e de um servidor OPC, projetado para receber e transmitir dados através da Web por meio da associação entre um software servidor OPC-DA e um middleware com função de gateway.

A teleoperação é fundamentada na associação do servidor de streaming de vídeo com o serviço de comandos DNC (Comando Numérico Distribuído) para operação remota da máquina via internet por meio de requisições HTTP com scritps CGI (common gateway interface). É possível considerar que funções do servidor OPCWeb também se enquadram entre os serviços de teleoperação. Esse servidor foi projetado para supervisionar os controles e intervir na operação da máquina-ferramenta, a exemplo da movimentação da torre através dos eixos da máquina. 
Foi construída uma interface cliente Web que integra as atividades fornecidos pelos servidores em um mesmo ecrã, desenvolvido essencialmente em linguagem HTML com CSS (Cascade Style Sheet) e Javascript utilizando plugin JQuery e metodologia Ajax.

As funções disponibilizadas com a arquitetura de implementação representam o mapeamento virtual de recursos importantes da máquina-ferramenta. A máquina-ferramenta CNC pode ser monitorada e sofrer atuação via internet. $\mathrm{O}$ sistema disponibiliza dados em formato universal, como o XML, o que garante maior integração vertical e horizontal em um sistema de produção. Apenas uma infraestrutura de internet robusta com uma largura de banda que garanta elevadas taxas de transmissão e recepção de dados permitirá a realização do potencial da aplicação em operar em tempo real. Os dados fornecidos pelo sistema, em propostas de desenvolvimentos futuros, podem ser salvos em bases de dados tratados a fim de apoiar tomadas de decisões mais precisas relacionadas à fabricação. Esse conjunto de atributos posiciona o sistema como uma aplicação para Internet de Serviços (IoS), e portanto, na Indústria 4.0.

Durante o trabalho de implementação houve a necessidade de aproveitar os benefícios de um variado número de linguagens de programação. Começando pelo servidor de monitoramento, utilizou-se da versatilidade e robustez da linguagem C\# na plataforma .Net para a programação do adaptador do CNC para o agente MTConnect. Este último teve seu código fonte escrito em C++, também na Microsoft .Net Framework, que foi estudado antes da sua instalação e configuração. O servidor de supervisão OPC, chamado de OPCWeb, contou com um módulo gateway que teve duas versões, uma em Web Service RESTful desenvolvido em Java com framework Jersey associado a API JOPCClient, e a outra com scripts desenvolvidos em Python utilizando mecanismo CGI. Os serviços de teleoperação utilizados na implementação computacional da arquitetura, em sua maioria, não foram necessariamente desenvolvidos, mas foram recursos herdados de outro projeto e sofreram ajustes para a sua implementação neste trabalho. Entre os serviços herdados de projetos anteriores, há o servidor de streaming de vídeo (WebCam) (Álvares, 2005) que emprega Applets Java e os scripts CGI para o servidor de comandos DNC (WebCNC) (Álvares, 2005), ambos são parte do serviço de teleoperação.

O cliente Web para integração e teste representado na Figura 6 é desenvolvido em HTML, utilizando CSS, PHP e JavaScript com JQuery. Na programação desse módulo optou-se pelo uso de linguagens interpretadas (não compiladas), mais leves, a fim de demonstrar a versatilidade da arquitetura implementada.

A interface Web cliente assume a função de elemento integrador dos servidores implementados da arquitetura do sistema, agregando controles que fazem requisições e recebem respostas na forma de dados e informações através dos servidores MTConnect, OPCWeb, WebCam e WebCNC. Todos esses serviços são reunidos no cliente web desenvolvido para monitoramento e teleoperação através da internet utilizando um software browser. Esse cliente, que é apresentado na Figura 6, é formado pelos seguintes componentes:

(A) Botões representando teclas do painel de operações do CNC do centro de torneamento. Esses botões estão vinculados as requisições de escrita (HTTP/PUT) no servidor OPCWeb. As teclas representadas na página Web são: Auto, Edit, MDI, JOG, Single Block, Block Delete, Dry Run, Prog Test, -X, +X, -Z, +Z, Cycle Start, Cycle Stop, Coolant ON, Coolant OFF, Coolant Auto e Reset.

(B) Painel representado os LEDs das teclas do CNC e outros tipos de sinais, formado por imagens que alteram de cor, conforme o estado dos controles se alteram no CNC. Sinal verde representa uma tecla ativada, alarme acionado ou a porta do centro de torneamento fechada. 
(C) Quadro para lançamento do streaming de dados resultante da comunicação com o servidor MTConnect, e que é acionado através do clique na tecla Start Streaming.

(D) Opções de comando remoto DNC selecionáveis através de botões de radio.

(E) Área para seleção de câmeras e exibição de imagens do processo provenientes do servidor WebCam.

(F) Painel para a exibição de mensagens ou alertas do CNC.

(G) Área para a exibição da lista de programas NC gravados na memória do controlador.
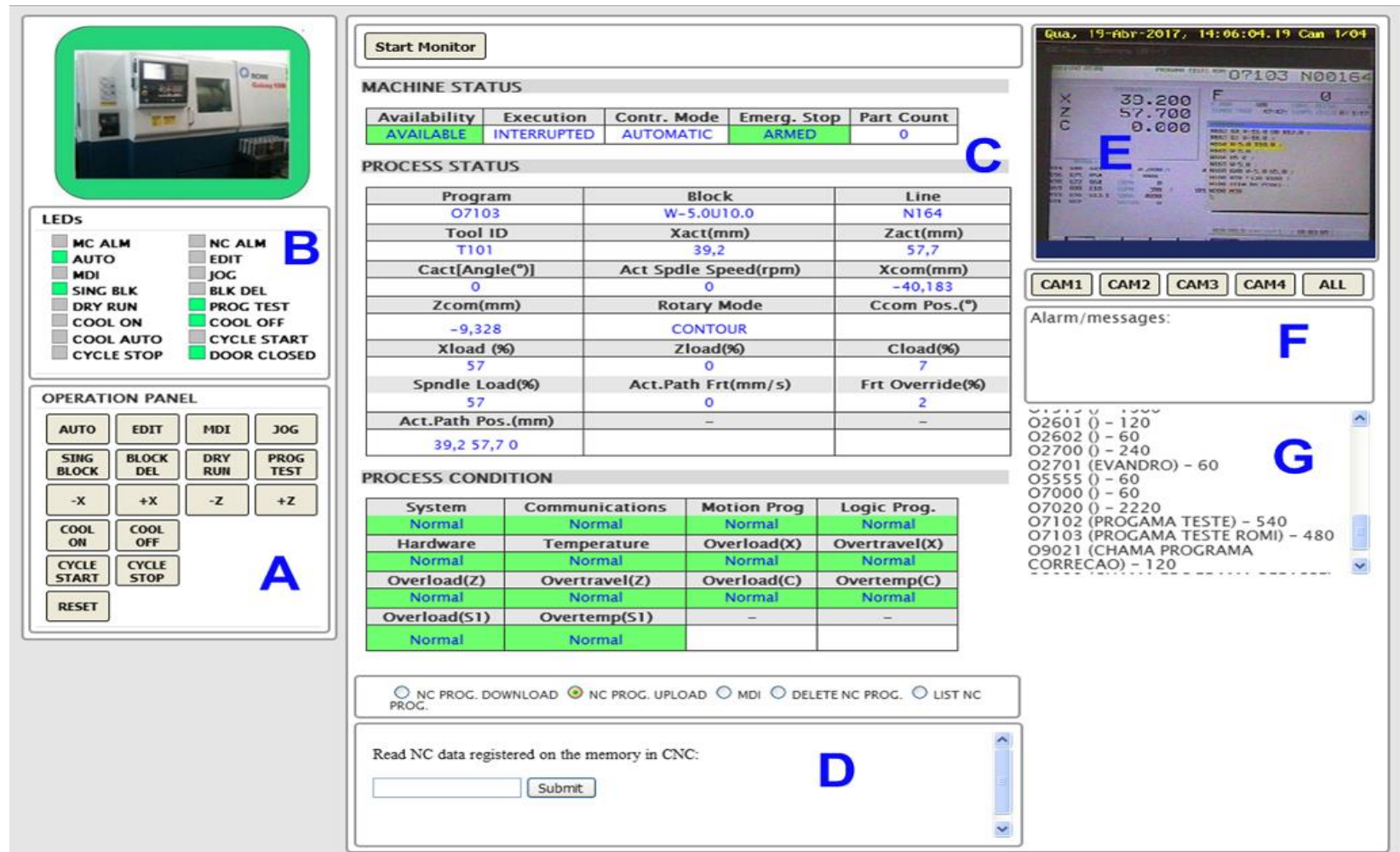

Figura 6: Cliente Web - Interface gráfica de monitoramento e teleoperação.

Fonte: Elaborado pelos autores (2017)

O sistema desenvolvido recebeu o nome de CyberDNC e como um sistema de apoio a manufatura compõe o grupo das aplicações para a Indústria 4.0 devido as características dos servidores que incorpora, na forma de serviços, e pelo potencial de novos desenvolvimentos que sugere a partir de sua arquitetura.

A capacidade de monitorar dados de um processo de fabricação transmitidos em um formato universal favorece uma maior integração vertical e horizontal em uma empresa de manufatura. Em futuras aplicações baseadas na arquitetura do framework, dados capturados podem ser salvos em bancos de dados que possibilitarão que análises desses dados apoiem tomadas de decisões mais efetivas. $\mathrm{O}$ uso de teleoperação através da internet identifica o sistema implementado entre as aplicações de telepresença baseadas na nuvem citadas por Kagermann et al. (2013).

O trabalho de teste e validação do sistema resultante da implementação do framework foi efetuado utilizando um centro de torneamento modelo Galaxy 15M da Romi, com CNC Fanuc 18iTa , que é o elemento físico fundamental para a condução do trabalho de projeto, desenvolvimento, teste e validação da proposta deste trabalho. 
O procedimento de teste e validação adotado foca na demonstração de funcionamento do sistema com base em casos de uso, tendo como referência na comunidade acadêmica trabalhos relacionados ao desenvolvimento de metodologias no âmbito da manufatura eletrônica (Álvares, (2005), Benavente (2007)).

Os testes foram divididos em avaliações por servidor, iniciando pelo servidor de monitoramento MTConnect, em que é verificada a capacidade do Agente em conectar-se com diferentes clientes Web baseados em PC, através de um browser ou aplicações para dispositivos móveis. Nessa etapa o CNC da máquina-ferramenta foi ajustado para a execução simulada (Prog Test) de uma peça em modo automático, processando linha-por-linha (Single Block) do programa $\mathrm{NC}$.

A segundo parte dos testes com a interface de usuário do CyberDNC foi realizada com o centro de torneamento em modo manual $(\mathrm{Jog})$, a fim de que através da movimentação dos eixos da máquina manualmente um alarme fosse intencionalmente provocado, para que a transmissão de parâmetros de condição (Process Condition) da máquina pudesse ser testada. O resultado é apresentado na Figura 7. É possível perceber que com a execução do programa NC parado, o parâmetro Controller Mode foi atualizado para Manual, e o parâmetro Rotary Mode foi atualizado para Spindle.

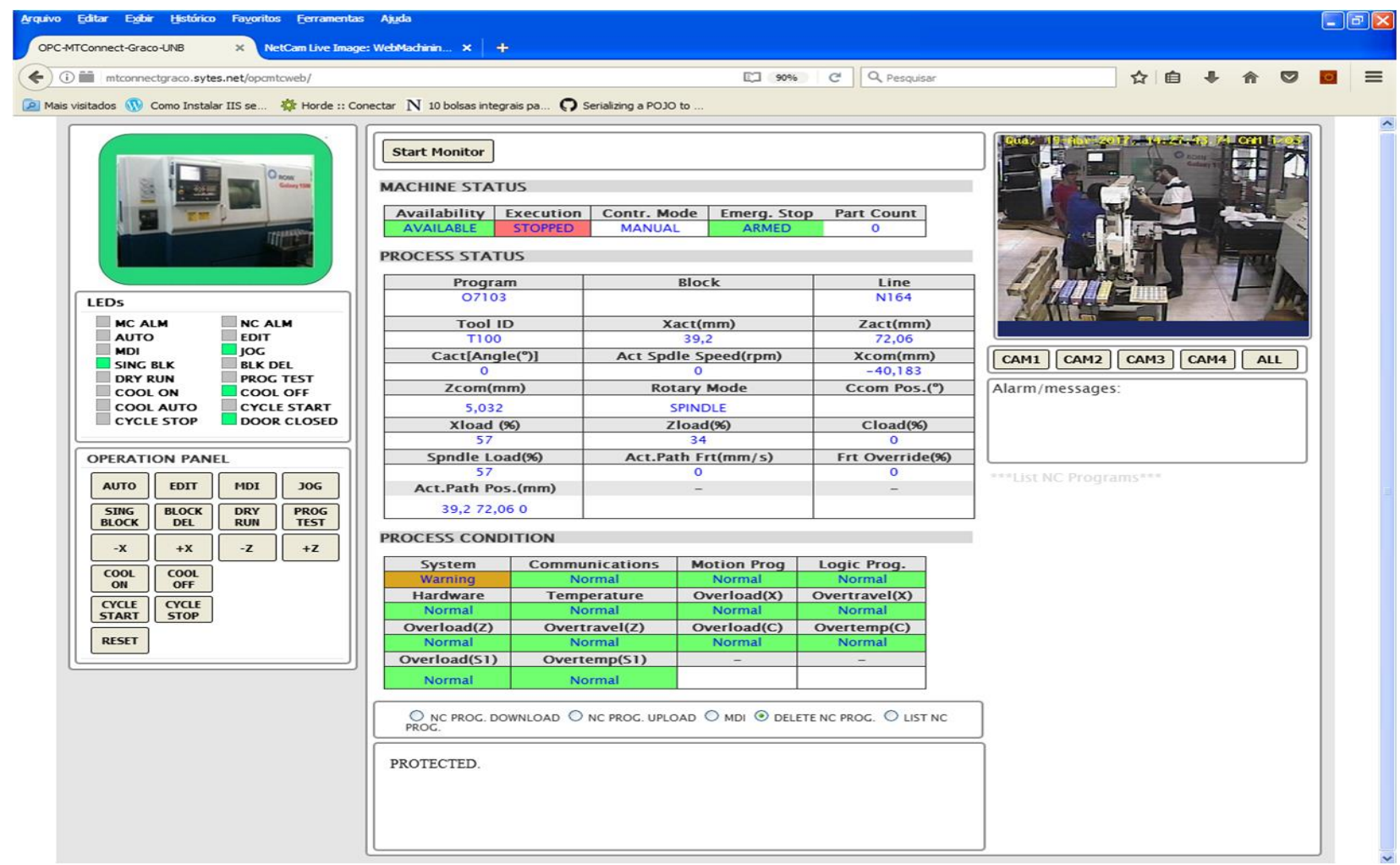

Figura 7: Teste da transmissão dos parâmetros de condição (Condition) pelo servidor MTConnect. Fonte: Elaborado pelos autores (2017)

Movimentando o eixo linear $\mathrm{Z}$ até o fim de curso com auxílio das teclas direcionais, o CNC produziu um alerta de sistema, conforme pode ser visualizado na Figura 7. Apesar representar uma avaliação relativamente simplificada, o resultado pode ser considerado representativo, pois no código fonte do Adaptador há uma classe exclusiva para o tratamento de condições.

As outras fases de teste coincidem com a avaliação dos outros servidores, como o servidor OPC para Web (OPCWeb), e os servidores WebCNC e WebCam de teleoperação. Todos esses 
servidores foram avaliados apenas com base no funcionamento do cliente web do CyberDNC, desenvolvido no âmbito deste trabalho.

Com auxílio dos controles de supervisão e comando remoto DNC da interface de usuário do CyberDNC, o parâmetro de velocidade rotacional (Spindle Speed) foi ajustado para três velocidades: 1000, 2000 e $3000 \mathrm{rpm}$. O resultado desses comandos foi transmitido para a aplicação com uma resolução de tempo nominal de 500 ms (milisegundos). A Figura 8 exibe o registro de quando a velocidade nominal do spindle foi alterada para $2000 \mathrm{rpm}$. Esses dados foram transmitidos para uma aplicação Android chamada GTMTC-Lite, um cliente MTConnect para dispositivos móveis. Esse teste foi uma constatação da versatilidade desse sistema ao transmitir e receber dados via internet independente de plataforma.

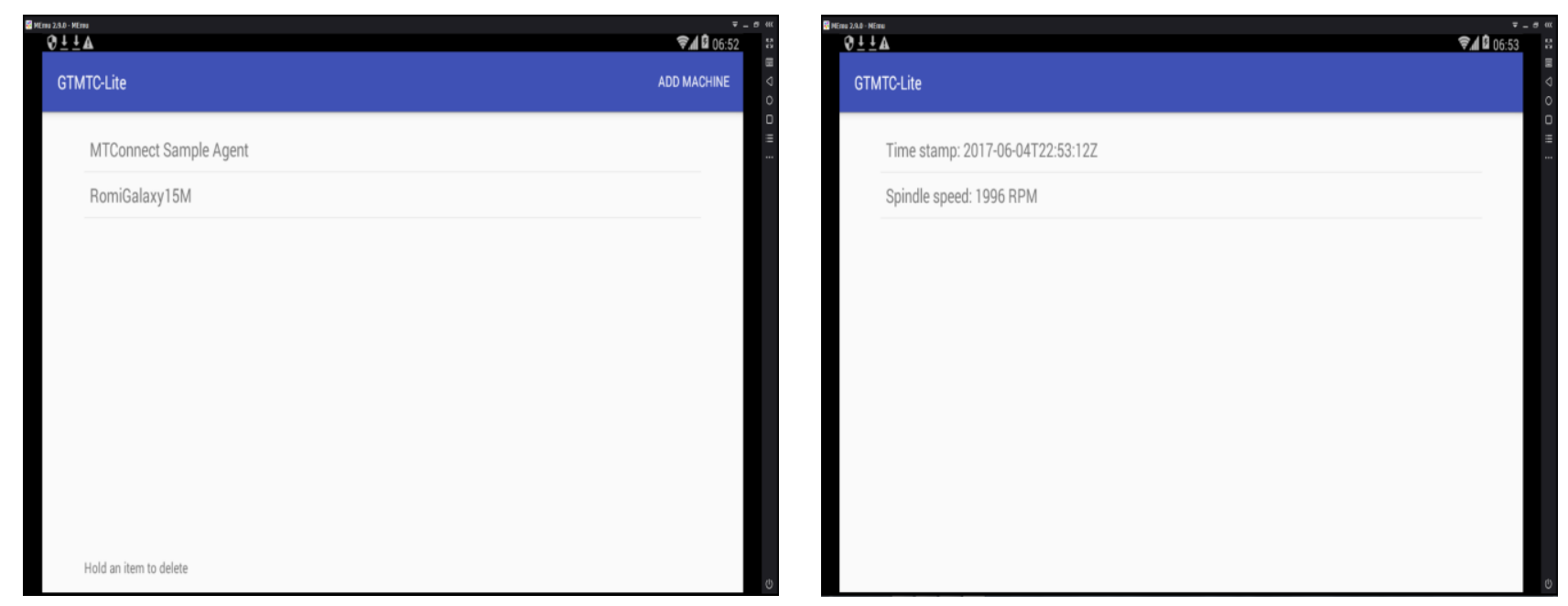

Figura 8: Cliente mobile GTMTC-Lite: streaming de dados da velocidade do spindle. Fonte: Elaborado pelos autores (2017)

As rotinas de teleoperação associadas a comando remoto (WebCNC) possuem um funcionamento baseado em requisições HTTP com programas CGI. É um serviço com uma complexidade menor em relação a outros serviços do framework e o tempo resposta não é um fator critico para a sua utilização, em comparação com atividades como monitoramento e supervisão. Esses fatores reduzem suas as chances de falha.

A GUI (Graphical User Interface) CyberDNC de teleoperação e monitoramento desenvolvida neste trabalho demonstrou bastante estabilidade durante os testes, associado ao fato de possuir uma interface amigável, organizada de forma lógica, o que favorece a sua usabilidade.

\section{CONCLUSÃO}

Este trabalho reuniu elementos do conceito de Manufatura Remota via internet, como a teleoperação, e das tecnologias de monitoramento disponíveis, associados às propostas tecnológicas para a manufatura da quarta revolução industrial (Indústria 4.0) com o propósito de conceber uma arquitetura de um framework que integrasse monitoramento, supervisão e teleoperação através da internet e estivesse em conformidade com esse novo paradigma da indústria de manufatura.

Com o propósito de fornecer uma visão ampla das funções previstas na arquitetura foi empregada a modelagem IDEF0. Este recurso deu ao framework um aspecto modular em que cada elemento nos diferentes níveis desse diagrama correspondesse a um serviço ou atividade a ser implementada. A arquitetura geral definida pelo IDEF0 auxiliou na definição das necessidades dos clientes do projeto, informações básicas para iniciar o processo de projeto axiomático. 
$\mathrm{O}$ uso do projeto axiomático para projeto detalhado do sistema possibilitou que fosse definida uma sequência de implementação. A implementação do serviço de monitoramento da arquitetura envolveu uma adequação dos recursos disponíveis em termos equipamentos e software, como a API e driver de comunicação com CNC Fanuc 18i, o Focas 1, que é compatível apenas com sistemas operacionais Windows, em versão não superior ao Windows XP. Foi necessário conciliar essas restrições com fato de viabilizar a operação de um serviço em que a velocidade de processamento e transmissão de dados são fatores-chave que influenciam na qualidade dos dados fornecidos.

O procedimento de teste do serviço MTConnect envolveu diferentes clientes Web acessando o Agente. Nesse teste de conectividade verificou-se que mais de um cliente conectou-se ao software e dele requisitou e recebeu como resposta o streaming com dados de fabricação provenientes do CNC Fanuc 18i-Ta. Esse teste validou o sistema como uma aplicação multiplataformas.

Neste trabalho, um dos desafios foi desenvolver um serviço em que um servidor OPC estivesse acessível via Internet e possuísse características que funcionalmente fossem similares as de um servidor OPC-UA, que é um padrão declarado como que reúne os atributos para um padrão da Indústria 4.0. Para isso, foram propostas duas soluções alternativas, a primeira envolve o uso de um Web Service RESTful como elemento gateway entre o servidor OPC e o cliente na Web. A segunda solução envolveu a utilização da API OpenOPC para Python, em que a comunicação com o servidor OPC e, consequentemente, com o CLP do centro de torneamento, baseia-se em requisições HTTP associadas a programas escritos em Python que são executados através de mecanismo CGI. Essa segunda alternativa foi a de implementação mais rápida e prática, e também a que garantiu o adequado funcionamento do serviço de supervisão do sistema.

No que se refere à teleoperação, a maioria dos módulos de software foram herdados de projetos anteriores, como os programas CGI utilizada na execução de comandos DNC remotamente, e o servidor de imagem (WebCam) da FMC. Nas funções implementadas usando mecanismo CGI o tempo de resposta não é considerado um fator crítico, de forma que as limitações desse protocolo nesse ponto não afetaram a usabilidade do sistema.

\section{AGRADECIMENTOS}

CNPQ, CAPES e Universidade de Brasília

\section{REFERÊNCIAS}

ALBERT, M. Seven things to know about the internet of things and industry 4.0. Modern Machine Shop Magazine, 2015. Disponível em: < https://www.mmsonline.com/articles/7-things-to-know-about-the-internet-of-things-andindustry-40>. Acessado em: 01.2017.

ÁlVARES, Alberto; FERREIRA, João; SHIMABUKURO, Leonardo; FERNANDES, Carlos. Um Sistema de Telemanufatura Baseado na Web Orientado ao Processo de Oxicorte In: ENCONTRO NACIONAL DE ENGENHARIA DE PRODUÇÃ̃, 22, 2002, Curitiba. Anais..., Curitiba: Abepro, 2002.

ÁLVARES, Alberto; SILVA, Fábio; FERREIRA, João. Webturning: Teleoperação de um centro de torneamento via internet In: Congresso Brasileiro de Engenharia de Fabricação, 3, 2005, Joinville. Anais.... Joinville: ABCM, 2005.

ÁLVARES, A. J. Uma metodologia para integração CAD/CAPP/CAM voltada para manufatura remota de peças rotacionais baseada na internet. Tese( Doutorado em Engenharia Mecânica) - Programa de Pós-graduação em Engenharia Mecânica, Universidade Federal de Santa Catarina, Florianópolis, 2005.

ÁlVARES, Alberto; FERREIRA, João Webmachining: System for the design and manufacture of featurebased parts through the web. ABCM Symposium Series in Mechatronics, v.3, p. 688-700, 2008. 
BENAVENTE, J.C.T. Um sistema para o projeto e fabricação remota de peças prismáticas via internet. Dissertação (Mestrado em Engenharia Mecânica) - Programa de Pós-graduação em Engenharia Mecânica, Universidade Federal de Santa Catarina, Florianópolis. 2007.

BENAVENTE, J.C.T. Um sistema para o projeto e fabricação de peças mecânicas a distância via internet aderente à norma iso 14649 (STEP-NC). Tese (Doutorado em Engenharia Mecânica) - Programa de Pós-graduação em Engenharia Mecânica, Universidade Federal de Santa Catarina, Florianópolis. 2011.

CHEN, S.; YIN, C.; LI, X. Implementation of mtconnect in machine monitoring system for CNCs. In: INTERNATIONAL CONFERENCE ON ENTERPRISE SYSTEMS (ES), 5, 2017, Beijing. Proceedings... Beijing: IEEE, 2017, p. 70-75.

FERNANDES, R.F; TORRISI, N. M.; BRANDÃO, D. Remote tuning of industrial controllers using CyberOPC technology. In: IEEE INTERNATIONAL CONFERENCE ON INDUSTRIAL INFORMATICS (INDIN), 7, 2009, Cardiff. Proceedings... Cardiff: IEEE, 2009, p. 750-756.

GUBBI, J.; BUYYA, R.; MARUSIC, S.; PALANISWAMI, M. Internet of things (IoT) : A vision, architectural elements, and future directions. Future Generation Computer Systems, v.29, p.1645-1660, set. 2013.

KAGERMANN, H; WAHLSTER, W.; HELBIG, J. Recommendations for Implementing the Strategic Initiative Industrie 4.0: Final Report of the Industrie 4.0 Working Group, Berlin: Acatech, 2013.

KIM, S.-J. ; SUH, N.; KIM, S.-G. Design of software systems based on axiomatic design. Annals of the CIRP, v.40, p.165-170, 1991.

LEE, J. E-manufacturing : fundamental, tools, and transformation. Robotics and Computer-Integrated Manufacturing, v.19, p.501-507, dez. 2003.

LEE, X.; HU, J. Design and research of remote monitoring system based on OPC XML-DA In: INTERNATIONAL PRE-OLYMPIC CONGRESS ON COMPUTER SCIENCE, 1, 2008, China. Proceedings... 2008, p. 147-151.

MACDOUGALL, W. Industrie 4.0 - Smart Manufacturing for the Future. Berlin: Germany Trade \& Invest, 2014.

MAHNKE, W.; LEITNER, S.-H.; DAMM, M. OPC Unified Archtecture. Berlin: Springer-Verlag Berlin Heidelberg, 2009.

MICHALOSKI, J. L.; ZHAO, Y. F.; LEE, B. E; RIPPEY, W. G. Web-enabled, real-time, quality assurance for machining production systems. Procedia CIRP, v.10, p.332-339, 2013.

MONOSTORI, L. Cyber-physical production systems: Roots, expectations and R\&D challenges. Procedia CIRP, v.17, p. 9-13, 2015.

NOF, S. Y. Collaborative E-work and e-mfg: The state of the art and challenges for production and logistics managers In: IFAC Symposium on Information Control Problems in Manufacturing (INCOM), 11, 2004, Salvador. Anais...Salvador: International Federation of Automatic Control, 2004.

ROCKWELL-AUTOMATION. Making sense of e-manufacturing: A roadmap for manufacturers - Part 1, Industry White Paper, jan. 2001.

SAHIN, C.; BOLAT, E.D. Development of remote control and monitoring of web-based distributed OPC system. Computer Standards and Interfaces, v.31, p. 984-993, 2009.

SCIENTIFIC-AMERICAN. 21st century medicine: Cybersurgery, 2000. Disponível em: <www.pbs.org/safarchive/4_class/45_pguides/pguide_605/4565_cyber.html>. Acessado em: 03.2017.

SHI, J.; WAN, J.; YAN, H.; SUO, H. A survey of cyber-physical systems In: INTERNATIONAL CONFERENCE ON WIRELESS COMMUNICATIONS AND SIGNAL PROCESSING, 1, 2011, Naijing. Proceedings...___: IEEE, 2011.

SILVA E. J.; ABUJAMRA, R.C.; GIUFFRIDA, A.D.B.; REIS, V.R.A. Web based supervisory system for plunge grinding operation. ABCM Symposium Series in Mechatronics, v.5, p.859-868, 2012.

SOBEL, W. Mtconnect standard part 1- overview and protocol - 1.1.0 - final. MTConnect Institute, 2010.

SUH, N.; DO, S. Axiomatic design of software system. CIRP Annals - Manufacturing Technology, v.49, p. 95-100, 2000.

SUH, N.P. Axiomatic Design of Software . In: Axiomatic Design - Advances and Applications. 1. ed, New York: Oxford University Press, v.5, p. 239-298, 2001. 
TOGAY, C.; CANIAZ, E. S.; DOGRU, A. H. Rule based axiomatic design theory guidance for software development In: COMPUTER SOFTWARE AND APPLICATIONS CONFERENCE WORKSHOPS (COMPSACW), 36, 2012, Izmir. Proceedings... : IEEE, 2012, p.544-552.

VERMA, N. K.; DEV, R.; DHAR, N. K.; SINGH, D. J.; SALOUR, A.. Real-time remote monitoring of an air compressor using mtconnect standard protocol. In: IEEE INTERNATIONAL CONFERENCE ON PROGNOSTICS AND HEALTH MANAGEMENT (ICPHM), 1, 2017, Dallas. Proceedings... IEEE, 2017, p.109-116.

VIJAYARAGHAVAN, A.; DORNFELD, D. Automated energy monitoring of machine tools. CIRP Annals Manufacturing Technology, v.59, p. 21-24, 2010. 